\title{
Fuzzy Risk Analysis for a Production System Based on the Nagel Point of a Triangle
}

\author{
Handan Akyar \\ Department of Mathematics, Faculty of Science, Anadolu University, 26470 Eskisehir, Turkey \\ Correspondence should be addressed to Handan Akyar; hakyar@anadolu.edu.tr \\ Received 17 December 2015; Accepted 9 March 2016 \\ Academic Editor: Rosana Rodriguez-Lopez
}

Copyright ( 2016 Handan Akyar. This is an open access article distributed under the Creative Commons Attribution License, which permits unrestricted use, distribution, and reproduction in any medium, provided the original work is properly cited.

\begin{abstract}
Ordering and ranking fuzzy numbers and their comparisons play a significant role in decision-making problems such as social and economic systems, forecasting, optimization, and risk analysis problems. In this paper, a new method for ordering triangular fuzzy numbers using the Nagel point of a triangle is presented. With the aid of the proposed method, reasonable properties of ordering fuzzy numbers are verified. Certain comparative examples are given to illustrate the advantages of the new method. Many papers have been devoted to studies on fuzzy ranking methods, but some of these studies have certain shortcomings. The proposed method overcomes the drawbacks of the existing methods in the literature. The suggested method can order triangular fuzzy numbers as well as crisp numbers and fuzzy numbers with the same centroid point. An application to the fuzzy risk analysis problem is given, based on the suggested ordering approach.
\end{abstract}

\section{Introduction}

Recently, ordering and ranking fuzzy numbers and their comparisons have become the main research interest in fuzzy decision analysis and real world problems (e.g., see [1-10] and references therein).

Prominent methods for the ordering and ranking of fuzzy numbers have been published since the method for ranking fuzzy numbers was initially proposed by Jain (e.g., see $[2,3,10-25]$ and references therein). The most widely used approach for ordering and ranking triangular fuzzy numbers is based on the centroid of a triangle, proposed by Yager (see [12]). Yager's method considers only the horizontal coordinate of the centroid for the ranking, which lessens its effectiveness. Murakami et al. improved Yager's method using both coordinates of the centroid (see [10]). To overcome the shortcomings of the aforementioned centroid methods, S.-J. Chen and S.-M. Chen submitted a ranking method that uses the centroid point and the standard deviation of fuzzy numbers (see [3]). In [26], Nazirah and Daud wholly reviewed certain centroid index methods.

In [22], Lee and Chen propose a new method for fuzzy risk analysis based on fuzzy numbers with different shapes and deviations. In [14], Abbasbandy and Asady propose a sign distance method, where its drawbacks are treated in [15] by constructing a revised sign distance method. A new method is proposed by Ezzati et al. [20] that is a modification of Abbasbandy and Hajjari's method (see [23]) which can also be used for ranking symmetric fuzzy numbers. In [19], Ezzati et al. revise this method to overcome certain shortcomings.

Another approach for the ranking and ordering of fuzzy numbers is given by Akyar et al., based on the incenter of a triangle, which is the center of an inscribed circle (see $[2,18])$. However, it will be shown that this method has certain shortcomings, namely, the fact that the result of this method is sometimes inconsistent with human intuition. Düzce suggests a different method using the center of the nine-point circle of a triangle (see [21]). However, Düzce's method also has certain drawbacks, namely, the fact that this method cannot rank fuzzy numbers with the same centroid.

Recently, an improved method, which considers the areas of the positive side, the areas of the negative side, and the spreads of generalized fuzzy numbers as the ranking factors for ranking fuzzy numbers, has been given by Jiang et al. [24]. Another approach for the ranking of generalized fuzzy numbers with different left and right heights is given by Chutia et al. using integral values [25]. This method considers nonnormal $p$-norm trapezoidal fuzzy numbers as well as 
p-norm generalized fuzzy numbers with different left and right heights.

In this paper, the incenter method will be revised using the Nagel point of a triangle to increase efficiency. The Nagel point is the point of intersection of the line segments from the vertices of the triangle to the points of tangency of the opposite excircles [27]. In order to save most of the information of triangular fuzzy numbers, we associate these fuzzy numbers with triplets instead of real numbers. We will show that, unlike previously mentioned methods, we can efficiently order triangular fuzzy numbers by the proposed method.

The rest of this paper is organized as follows. In Section 2, fuzzy numbers, their fundamental properties, basic definitions, and theorems regarding the Nagel point of a triangle are reviewed. Next, in Section 3, we present a new method for ordering triangular fuzzy numbers based on the Nagel point of a triangle. In Section 4, we investigate reasonable properties of ordering triangular fuzzy numbers by the proposed method. In Section 5, numerical examples are given. We compare the results of the proposed method with other existing methods. An application to the fuzzy risk analysis problem of the proposed method is presented in Section 6. Finally, a conclusion is given in Section 7.

\section{Preliminaries}

2.1. Fuzzy Numbers. Here, certain essential concepts of fuzzy numbers and their basic properties will be given. For further information see $[28,29]$.

A fuzzy set $\widetilde{A}$ on a set $X$ is a function $\widetilde{A}: X \rightarrow[0,1]$. Commonly, the symbol $\mu_{\widetilde{A}}$ is used for the function $\widetilde{A}$, and it is said that the fuzzy set $\widetilde{A}$ is characterized by its membership function $\mu_{\widetilde{A}}: X \rightarrow[0,1]$ which associates each $x \in X$ with a real number $\mu_{\widetilde{A}}(x) \in[0,1]$. The degree to which $x$ belongs to $\widetilde{A}$ is interpreted by the value of $\mu_{\widetilde{A}}(x)$.

Let $\widetilde{A}$ be a fuzzy set on $X$. The support of $\widetilde{A}$ is given as

$$
S(\widetilde{A})=\left\{x \in X: \mu_{\widetilde{A}}(x)>0\right\},
$$

and the height $h(\widetilde{A})$ of $\widetilde{A}$ is defined as

$$
h(\widetilde{A})=\sup _{x \in X} \mu_{\widetilde{A}}(x) .
$$

The fuzzy set $\widetilde{A}$ is called a normal fuzzy set if $h(\widetilde{A})=1$.

Let $\widetilde{A}$ be a fuzzy set on $X$ and $\alpha \in[0,1]$. The $\alpha$-cut $(\alpha$-level set) of the fuzzy set $\widetilde{A}$ is given by

$$
[\widetilde{A}]^{\alpha}= \begin{cases}\left\{x \in X: \mu_{\widetilde{A}}(x) \geq \alpha\right\}, & \text { if } \alpha \in(0,1] \\ \operatorname{cl} S(\widetilde{A}), & \text { if } \alpha=0,\end{cases}
$$

where $\mathrm{cl}$ denotes the closure of sets.

A fuzzy set $\widetilde{A}$ on $\mathbb{R}^{n}$ is called a convex fuzzy set if its $\alpha$-cuts $[\widetilde{A}]^{\alpha}$ are convex sets for all $\alpha \in[0,1]$.

If $\widetilde{A}$ is a fuzzy set in $\mathbb{R}$, then $\widetilde{A}$ is called a fuzzy number if

(1) $\widetilde{A}$ is convex,

(2) $\widetilde{A}$ is normal,
(3) $\mu_{\widetilde{A}}$ is upper semicontinuous,

(4) the support of $\widetilde{A}$ is bounded.

Hereafter, lower case lettering such as $\widetilde{a}$ will be used to denote fuzzy numbers.

Generally, certain special types of fuzzy numbers, such as triangular, trapezoidal, and $L R$-fuzzy numbers, are used for real life applications (e.g., see [1-10] and references therein).

Let $L, R:[0,1] \rightarrow[0,1]$ be two continuous and increasing functions satisfying $L(0)=R(0)=0, L(1)=$ $R(1)=1$. Let $a_{0}^{-} \leq a_{1}^{-} \leq a_{1}^{+} \leq a_{0}^{+}$be real numbers. The fuzzy number $\widetilde{a}: \mathbb{R} \rightarrow[0,1]$ is an $L R$-fuzzy number if

$$
\tilde{a}(x)= \begin{cases}0 & \text { if } x<a_{0}^{-} \\ L\left(\frac{x-a_{0}^{-}}{a_{1}^{-}-a_{0}^{-}}\right) & \text {if } a_{0}^{-} \leq x<a_{1}^{-} \\ 1 & \text { if } a_{1}^{-} \leq x<a_{1}^{+} \\ R\left(\frac{a_{0}^{+}-x}{a_{0}^{+}-a_{1}^{+}}\right) & \text {if } a_{1}^{+} \leq x<a_{0}^{+} \\ 0 & \text { if } a_{0}^{+} \leq x .\end{cases}
$$

In particular, we get trapezoidal fuzzy numbers when the functions $L$ and $R$ are linear. Furthermore, if $a_{0}^{-}=a \leq a_{1}^{-}=$ $b=a_{1}^{+}=c \leq a_{0}^{+}=d$ and

$$
\widetilde{a}(x)= \begin{cases}0 & \text { if } x<a \\ \frac{x-a}{b-a} & \text { if } a \leq x<b \\ \frac{d-x}{d-b} & \text { if } b \leq x<d \\ 0 & \text { if } d \leq x\end{cases}
$$

then the fuzzy number $\tilde{a}$ is called a triangular fuzzy number. Then we can denote a triangular fuzzy number by a triplet $\left(a_{l}, a_{m}, a_{r}\right)\left(a_{l} \leq a_{m} \leq a_{r}\right)$ and we can write $\tilde{a}:=\left(a_{l}, a_{m}, a_{r}\right)_{T}$.

Let $\widetilde{a}$ and $\widetilde{b}$ be fuzzy numbers. Then the sum of fuzzy numbers $\tilde{a}$ and $\tilde{b}$ is defined as

$$
\mu_{\widetilde{a}+\tilde{b}}(z)=\sup _{z=x+y} \min \left\{\mu_{\tilde{a}}(x), \mu_{\tilde{b}}(y)\right\} .
$$

In particular, if $\tilde{a}=\left(a_{l}, a_{m}, a_{r}\right)_{T}$ and $\tilde{b}=\left(b_{l}, b_{m}, b_{r}\right)_{T}$ are triangular fuzzy numbers, then one can verify that

$$
\tilde{a}+\tilde{b}=\left(a_{l}+b_{l}, a_{m}+b_{m}, a_{r}+b_{r}\right)_{T} .
$$

The multiplication of fuzzy numbers $\widetilde{a}$ and $\widetilde{b}$ is also defined as

$$
\mu_{\tilde{a} \cdot \tilde{b}}(z)=\sup _{z=x \cdot y} \min \left\{\mu_{\tilde{a}}(x), \mu_{\tilde{b}}(y)\right\}
$$

Although $\tilde{a}$ and $\tilde{b}$ are triangular fuzzy numbers, their multiplication $\widetilde{a} \cdot \widetilde{b}$ is not a triangular fuzzy number. For convenience, we can express it as a triangular fuzzy number using two end points and one peak point. Therefore, for triangular fuzzy numbers $\tilde{a}=\left(a_{l}, a_{m}, a_{r}\right)_{T}$ and $\tilde{b}=\left(b_{l}, b_{m}, b_{r}\right)_{T}$ 


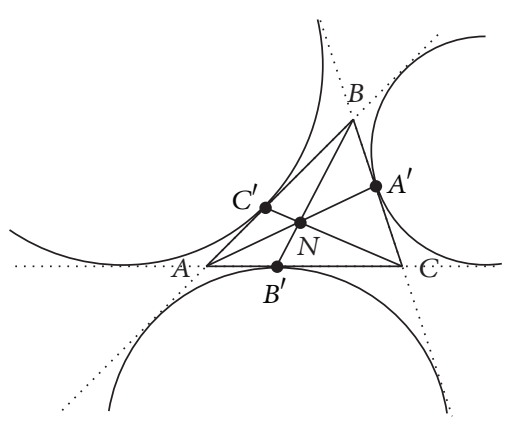

Figure 1: The Nagel point $N$ of a triangle $A B C$.

we define $\tilde{a} \cdot \tilde{b}=\left(a_{l} b_{l}, a_{m} b_{m}, a_{r} b_{r}\right)_{T}$. Similarly, we define $\tilde{a} / \widetilde{b}=\left(a_{l} / b_{l}, a_{m} / b_{m}, a_{r} / b_{r}\right)_{T}$.

The set of all triangular fuzzy numbers will be denoted by $\mathbb{T}$. In this paper, we will only consider triangular fuzzy numbers.

2.2. Nagel Point of a Triangle. The Nagel point $N$ of a triangle $A B C$ is defined as the point of intersection of the cevians $A A^{\prime}, B B^{\prime}$, and $C C^{\prime}$, where $A^{\prime}, B^{\prime}$, and $C^{\prime}$ are points where the excircles touch the sides (Figure 1). The existence of the Nagel point was proved in 1836 , by German mathematician C. H. Nagel.

Theorem 1 (Nagel Point Theorem [30]). The cevians joining the vertices of a triangle to the points of tangency of the opposite sides with the corresponding excircles are concurrent.

Note that, in Figure $1,|A B|+\left|B A^{\prime}\right|=|A C|+\left|C A^{\prime}\right|$. Hence, point $A^{\prime}$ is halfway-around the perimeter of the triangle from vertex $A$. Similarly, points $B^{\prime}$ and $C^{\prime}$ are also halfway-around vertices $B$ and $C$, respectively. Therefore, the Nagel Point Theorem is also named the Halfway-Around Theorem.

Given a triangle $A B C$, the trilinear coordinates of a point $P$, with respect to triangle $A B C$, are an ordered triple of numbers, each of which is proportional to the directed distance from $P$ to one of the side lines. Trilinear coordinates are usually denoted by $\alpha: \beta: \gamma$ (see Figure 2). Trilinear coordinates of the Nagel point are given as

$$
\csc ^{2}\left(\frac{\widehat{B A C}}{2}\right): \csc ^{2}\left(\frac{\widehat{A B C}}{2}\right): \csc ^{2}\left(\frac{\widehat{A C B}}{2}\right)
$$

or its equivalent:

$$
\frac{b+c-a}{a}: \frac{c+a-b}{b}: \frac{a+b-c}{c}
$$

where $a=|B C|, b=|C A|$, and $c=|A B|$ (see [31]).

If point $P$ has trilinear coordinates $\alpha: \beta: \gamma$, then the Cartesian coordinates of $P$ are calculated as follows:

$$
\begin{aligned}
P= & \frac{a \alpha}{a \alpha+b \beta+c \gamma} A+\frac{b \beta}{a \alpha+b \beta+c \gamma} B \\
& +\frac{c \gamma}{a \alpha+b \beta+c \gamma} C .
\end{aligned}
$$

Here, $a=|B C|, b=|C A|$, and $c=|A B|$.

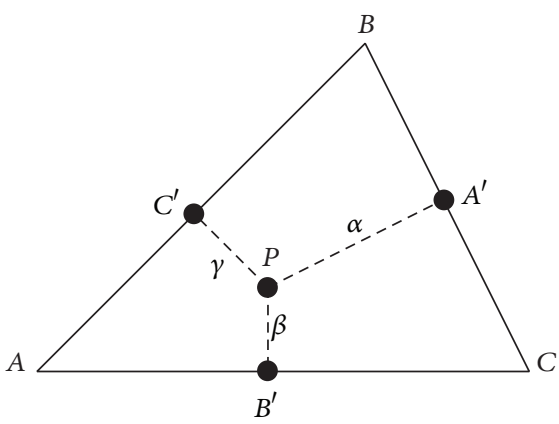

Figure 2: Trilinear coordinates $\alpha: \beta: \gamma$ of point $P$.

Proposition 2. Let $N$ be a Nagel point of triangle $A B C$. If $|B C| \rightarrow 0^{+}$then $N \rightarrow A$.

Proof. Let $N$ have the trilinear coordinates $\alpha: \beta: \gamma$. If $|B C| \rightarrow 0^{+}$, then by the definition of trilinear coordinates $\beta \rightarrow 0$ and $\gamma \rightarrow 0$. Using (11) we achieve the desired result.

\section{A New Method for Ordering Triangular Fuzzy Numbers}

Let $\tilde{a}=\left(a_{l}, a_{m}, a_{r}\right)_{T}$ be a triangular fuzzy number. Then this corresponds to the triangle $A B C$ with Cartesian coordinates $A\left(a_{l}, 0\right), B\left(a_{m}, 1\right)$, and $C\left(a_{r}, 0\right)$ in the Euclidean plane. Then we find

$$
\begin{aligned}
& a=|B C|=\sqrt{\left(a_{m}-a_{r}\right)^{2}+1}, \\
& b=|A C|=\left(a_{r}-a_{l}\right), \\
& c=|A B|=\sqrt{\left(a_{m}-a_{l}\right)^{2}+1} .
\end{aligned}
$$

Using (9) and (12), we find trilinear coordinates $\alpha: \beta: \gamma$ of Nagel point $N$ of triangle $A B C$ as follows:

$$
\begin{aligned}
& \alpha=\frac{a_{r}-a_{l}+\sqrt{\left(a_{m}-a_{l}\right)^{2}+1}-\sqrt{\left(a_{m}-a_{r}\right)^{2}+1}}{\sqrt{\left(a_{m}-a_{r}\right)^{2}+1}}, \\
& \beta=\frac{\sqrt{\left(a_{m}-a_{l}\right)^{2}+1}+\sqrt{\left(a_{m}-a_{r}\right)^{2}+1}-a_{r}+a_{l}}{a_{r}-a_{l}}, \\
& \gamma=\frac{\sqrt{\left(a_{m}-a_{r}\right)^{2}+1}+a_{r}-a_{l}-\sqrt{\left(a_{m}-a_{l}\right)^{2}+1}}{\sqrt{\left(a_{m}-a_{l}\right)^{2}+1}} .
\end{aligned}
$$

Therefore, for all $\tilde{a} \in \mathbb{T}$ by means of the given method one can always get a point in the Euclidean plane.

Using (11) the Cartesian coordinates of $N_{\tilde{a}}=\left(x_{\tilde{a}}, y_{\tilde{a}}\right)$ for $\tilde{a} \in \mathbb{T}$ can be calculated as follows:

$$
\begin{aligned}
N_{\tilde{a}} & =\left(x_{\tilde{a}}, y_{\tilde{a}}\right) \\
& =\left(\frac{a \alpha a_{l}+b \beta a_{m}+c \gamma a_{r}}{a \alpha+b \beta+c \gamma}, \frac{b \beta}{a \alpha+b \beta+c \gamma}\right) .
\end{aligned}
$$


Different triangular fuzzy numbers may have the same Nagel point. Indeed let $\widetilde{a}=(0,0,2 \sqrt{2})_{T}, \widetilde{b}=(1,2,3)_{T}$, and $\tilde{c}=(4-2 \sqrt{2}, 4,4)_{T}$; then using (9) and (11) we get the same Nagel point

$$
N_{\tilde{a}}=N_{\tilde{b}}=N_{\tilde{c}}=(2,3-2 \sqrt{2}) .
$$

Therefore, the correspondence between triangular fuzzy numbers and their Nagel points is not one to one.

Let $\tilde{a}=\left(a_{l}, a_{m}, a_{r}\right)_{T}$ and $\widetilde{b}=\left(b_{l}, b_{m}, b_{r}\right)_{T}$ be two different triangular fuzzy numbers, such that $a_{m}=b_{m}$. If $N_{\tilde{a}}=N_{\tilde{b}}$ then it is obvious that $\widetilde{a}=\widetilde{b}$. Hence, the correspondence

$$
\begin{aligned}
& \mathcal{N}(\cdot): \mathbb{T} \longrightarrow\left\{(x, y, z) \in \mathbb{R}^{3}: 0<y<1\right\}, \\
& \mathcal{N}(\widetilde{a})=\left(x_{\widetilde{a}}, y_{\widetilde{a}}, a_{m}\right)
\end{aligned}
$$

is one to one. Here, $N_{\tilde{a}}=\left(x_{\tilde{a}}, y_{\widetilde{a}}\right)$ and $\widetilde{a}=\left(a_{l}, a_{m}, a_{r}\right)_{T}$.

Using lexicographical order, we can now order triangular fuzzy numbers by the function $\mathcal{N}$ defined by (16); that is, for triangular fuzzy numbers $\widetilde{a}=\left(a_{l}, a_{m}, a_{r}\right)_{T}$ and $\widetilde{b}=\left(b_{l}, b_{m}, b_{r}\right)_{T}$

$$
\widetilde{a}<\widetilde{b} \quad \text { iff } \mathcal{N}(\widetilde{a})<_{L} \mathcal{N}(\widetilde{b}),
$$

where the symbol $<_{L}$ denotes the lexicographical order, and it is defined as follows:

$$
\begin{aligned}
\left(x_{1}, x_{2}, x_{3}\right)<_{L}\left(y_{1}, y_{2}, y_{3}\right) & \\
\Longleftrightarrow & (\exists m=1,2,3)(\forall i<m)\left(x_{i}=y_{i}\right) \\
& \wedge\left(x_{m}<y_{m}\right) .
\end{aligned}
$$

Obviously, $\left(x_{1}, x_{2}, x_{3}\right)<_{L}\left(y_{1}, y_{2}, y_{3}\right)$ if and only if $x_{1}<y_{1}$ or $\left(x_{1}=y_{1}\right.$ and $\left.x_{2}<y_{2}\right)$ or $\left(x_{1}=y_{1}\right.$ and $x_{2}=y_{2}$ and $\left.x_{3}<y_{3}\right)$.

In view of Proposition 2, we get the following result.

Proposition 3. Let $\delta_{1}, \delta_{2}$ be arbitrarily small positive real numbers and let $\tilde{a}=\left(a_{m}-\delta_{1}, a_{m}, a_{m}+\delta_{2}\right)_{T}$ be a triangular fuzzy number. Then

$$
\lim _{\delta_{1}, \delta_{2} \rightarrow 0^{+}} N_{\tilde{a}}=\left(a_{m}, 1\right) .
$$

Therefore, if a triangular fuzzy number $\widetilde{a}=\left(a_{l}, a_{m}, a_{r}\right)_{T}$ is a crisp number, that is, $a_{l}=a_{m}=a_{r}$, then we can assume that $N_{\tilde{a}}=\left(a_{m}, 1\right)$. Hence, using the proposed method, we can also order crisp numbers. Furthermore, since the function defined by (16) is one to one, the proposed method is discriminative.

Example 4. Let $\widetilde{a}=(0,2,4)_{T}, \widetilde{b}=(1,4,5)_{T}$, and $\widetilde{c}=(1,2,3)_{T}$ be triangular fuzzy numbers and let $\widetilde{d}=(5,5,5)_{T}$ be a crisp number (see Figure 3). Then using (14) and Proposition 3 we get

$$
\begin{aligned}
& N_{\tilde{a}}=(2,0.0557), \\
& N_{\tilde{b}}=(2.2519,0.0672), \\
& N_{\tilde{c}}=(2,0.1716), \\
& N_{\tilde{d}}=(5,1) .
\end{aligned}
$$

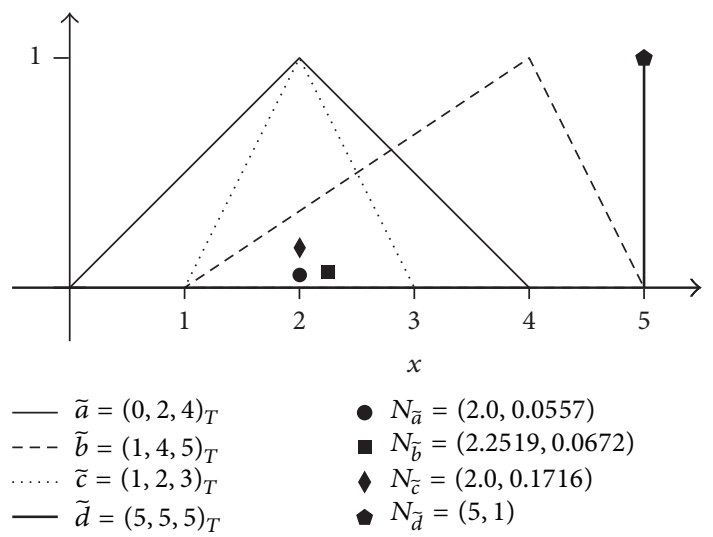

FIgURE 3: Triangular fuzzy numbers with their Nagel points in Example 4.

Since $\mathcal{N}(\widetilde{a})=(2,0.0557,2), \mathcal{N}(\widetilde{b})=(2.2519,0.0672,4)$, $\mathcal{N}(\tilde{c})=(2,0.1716,2)$, and $\mathcal{N}(\tilde{d})=(5,1,5)$, we have

$$
\begin{aligned}
(2,0.0557,2) & <{ }_{L}(2,0.1716,2)<_{L}(2.2519,0.0672,4) \\
& <_{L}(5,1,5)
\end{aligned}
$$

and we find

$$
\tilde{a}<\tilde{c} \prec \tilde{b} \prec \tilde{d}
$$

as expected.

\section{Reasonable Ordering Properties}

In [32, 33], Wang and Kerre propose certain axioms as reasonable properties to determine the rationality of an order method. In this section, it will be shown that the proposed method satisfies Wang and Kerre's reasonable properties.

Let $\tilde{a}, \tilde{b} \in \mathbb{T}$. If $\tilde{a}=\widetilde{b}$ or $\widetilde{a} \prec \widetilde{b}$, then we write $\widetilde{a} \leq \widetilde{b}$. It is clear that when the proposed method is applied on the set of all triangular fuzzy numbers $\mathbb{T}$, one of the following is true for $(\widetilde{a}, \widetilde{b}) \in \mathbb{T} \times \mathbb{T}: \widetilde{a}<\widetilde{b}, \widetilde{b}<\widetilde{a}, \widetilde{a}=\widetilde{b}$.

Now, we investigate the following axioms defined as reasonable properties of ordering triangular fuzzy numbers for the proposed method.

(1) It is clear that, for an arbitrary finite subset $U$ of $\mathbb{T}$ and $\tilde{a} \in U, \widetilde{a} \leq \widetilde{a}$ by the proposed method on $U$.

(2) Since lexicographical order is a total order relation, for an arbitrary finite subset $U$ of $\mathbb{T}$ and $(\widetilde{a}, \widetilde{b}) \in U \times$ $U, \widetilde{a} \preceq \widetilde{b}$ and $\widetilde{b} \preceq \widetilde{a}$ by the proposed method on $U$, we have $\tilde{a}=\widetilde{b}$ by the proposed method on $U$.

(3) Similarly, since lexicographical order is a total order relation, for an arbitrary finite subset $U$ of $\mathbb{T}$ and $(\widetilde{a}, \widetilde{b}, \widetilde{c}) \in U \times U \times U, \widetilde{a} \leq \widetilde{b}$ and $\widetilde{b} \leq \widetilde{c}$ by the proposed method on $U$, we have $\widetilde{a} \leq \widetilde{c}$ by the proposed method on $U$.

(4) For an arbitrary finite subset $U$ of $\mathbb{T}$ and $(\widetilde{a}, \widetilde{b}) \in U \times U$,

$$
\sup S(\widetilde{a})<\inf S(\widetilde{b}),
$$


we have $\widetilde{a} \prec \widetilde{b}$ by the proposed method on $U$, where $S(\widetilde{a})$ denotes the support of triangular fuzzy number $\widetilde{a}$ defined by (1). Indeed, if $\widetilde{a}=\left(a_{l}, a_{m}, a_{r}\right)_{T}$ and $\widetilde{b}=$ $\left(b_{l}, b_{m}, b_{r}\right)_{T}$ then $\sup S(\widetilde{a})=a_{r}$ and inf $S(\widetilde{b})=b_{l}$. By the definition of a Nagel point, we get $x_{\tilde{a}}<x_{\tilde{b}}$, where $x_{\tilde{a}}$ and $x_{\tilde{b}}$ are horizontal coordinates of the Nagel points of $\widetilde{a}$ and $\widetilde{b}$, respectively.

This axiom means that if two triangular fuzzy numbers have separate supports, then the fuzzy number with support on the right is at least as good as the one with support on the left.

(5) Let $U$ and $U^{\prime}$ be arbitrary sets of triangular fuzzy numbers and let $\tilde{a}$ and $\tilde{b}$ be in $U \cap U^{\prime}$. Then it is clear that $\widetilde{a}<\widetilde{b}$ by the proposed method on $U$ if and only if $\widetilde{a}<\widetilde{b}$ by the proposed method on $U^{\prime}$.

(6) Let $\widetilde{a}, \widetilde{b}$, and $\widetilde{c}$ be elements of $\mathbb{T}$. Then by the definition of the sum of fuzzy numbers $\widetilde{a}+\widetilde{c} \in \mathbb{T}$ and $\widetilde{b}+\widetilde{c} \in \mathbb{T}$. If $\widetilde{a}<\widetilde{b}$ by the proposed method on $\{\tilde{a}, \widetilde{b}\}$, then $\tilde{a}+\tilde{c}<$ $\widetilde{b}+\tilde{c}$ by the proposed method on $\{\widetilde{a}+\widetilde{c}, \widetilde{b}+\widetilde{c}\}$ except for the case in which $\widetilde{a}$ and $\widetilde{b}$ are isosceles triangles with the same peak point. Indeed, if $\widetilde{a}=(-2,0,2)_{T}$, $\widetilde{b}=(-1,0,1)_{T}$, and $\widetilde{c}=(1,2,4)_{T}$ then it is easy to find that $N_{\tilde{a}}=(0,9-4 \sqrt{5})$ and $N_{\tilde{b}}=(0,3-2 \sqrt{2})$. Hence, we get $\widetilde{a} \prec \widetilde{b}$. However, since $N_{\tilde{a}+\tilde{c}}=(2.962,0.020)$ and $N_{\tilde{b}+\tilde{c}}=(2.925,0.038)$ we obtain $\widetilde{b}+\widetilde{c} \prec \widetilde{a}+\widetilde{c}$.

(7) Let $\widetilde{a}, \widetilde{b}$, and $\widetilde{c}$ be triangular fuzzy numbers, such that $\tilde{c}=\left(c_{l}, c_{m}, c_{r}\right)_{T}$ and $c_{l}, c_{m}$, and $c_{r}$ are nonnegative real numbers. Then $\widetilde{a} \preceq \widetilde{b}$ by the proposed method on $\{\tilde{a}, \tilde{b}\}$ implies that $\tilde{a} \cdot \widetilde{c} \leq \widetilde{b} \cdot \widetilde{c}$ by the proposed method on $\{\widetilde{a} \cdot \widetilde{c}, \widetilde{b} \cdot \widetilde{c}\}$ except for the case in which $\widetilde{a}$ and $\widetilde{b}$ are isosceles triangles with the same peak point.

\section{Numerical Examples}

In this section, we initially give a simple example to show that the proposed method overcomes the shortcomings of the method of Akyar et al. (see [18]).

Example 5. Consider two triangular fuzzy numbers $\widetilde{a}=$ $(0,0.4,0.7)_{T}$ and $\widetilde{b}=(0.2,0.3,0.5)_{T}$ (see Figure 4). According to the method of Akyar et al. [18], the coordinates of the incenters of triangles corresponding to triangular fuzzy numbers $\widetilde{a}$ and $\widetilde{b}$ are $(0.6184,0.7519)$ and $(0.7135,0.8709)$, respectively. Hence, in view of this method, we get $\widetilde{a} \prec \widetilde{b}$, but this is not consistent with human intuition.

However, using the proposed method we get from (14)

$$
\begin{aligned}
& N_{\tilde{a}}=(0.3669,0.5037), \\
& N_{\tilde{b}}=(0.3148,0.7419) .
\end{aligned}
$$

Therefore, we have $\mathcal{N}(\widetilde{a})=(0.3669,0.5037,0.4)$ and $\mathscr{N}(\widetilde{b})=$ $(0.3148,0.7419,0.3)$. Since

$$
(0.3148,0.7419,0.3)<_{L}(0.3669,0.5037,0.4)
$$

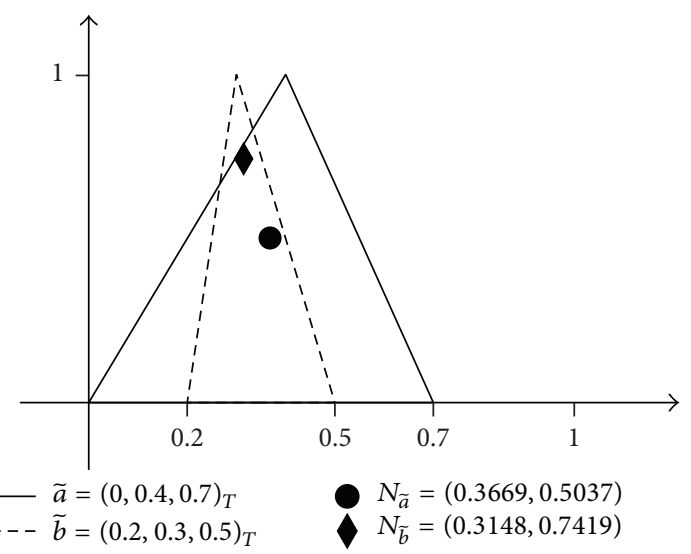

FIgURE 4: Triangular fuzzy numbers with their Nagel points in Example 5.

we find $\widetilde{b}<\widetilde{a}$ which is reasonable and similar to the results of the aforementioned methods (see $[15,19,21,24,25]$ ).

Thus, the proposed method overcomes the shortcoming of the method of Akyar et al. in this example.

Now, to compare the proposed method with certain existing methods, we use seven sets of triangular fuzzy numbers. Four of these are adopted from [3, 22]. Note that we set $\alpha=0.5$ in the method of Chutia et al. [25]. The sets are shown in Figure 5, and a comparison of the results for different methods is shown in Table 1.

From Table 1, we can see the drawbacks of the existing methods, described as follows:

(1) In Set 1, Set 4, and Set 6 of Figure 5, all the listed methods achieve the same order as well as the proposed method.

(2) In Set 2 of Figure 5, the method of Düzce and Chutia et al. is not discriminative. For $p=1$ the revised sign distance method of Abbasbandy et al. is not discriminative and for $p=2,3,4$ as it achieves a counterintuitive order. However the methods of Lee and Chen, S.-J. Chen and S.-M. Chen, Akyar et al., and Ezzati et al. and the proposed method obtain the same order.

(3) In Set 3 of Figure 5, all listed methods achieve the same order except for the method of Ezzati et al.

(4) In Set 5 of Figure 5, the method of S.-J. Chen and S.M. Chen achieves a counterintuitive order, while the proposed method and the other listed methods obtain the same order.

(5) In Set 7 of Figure 5, the methods of S.-J. Chen and S. M. Chen, Abbasbandy et al., and Akyar et al. achieve a counterintuitive order. However, by the proposed method we have overcome this shortcoming and obtain the same order as the other listed methods.

Thus, other listed methods have certain drawbacks, such as the result order being inconsistent with human intuition; 

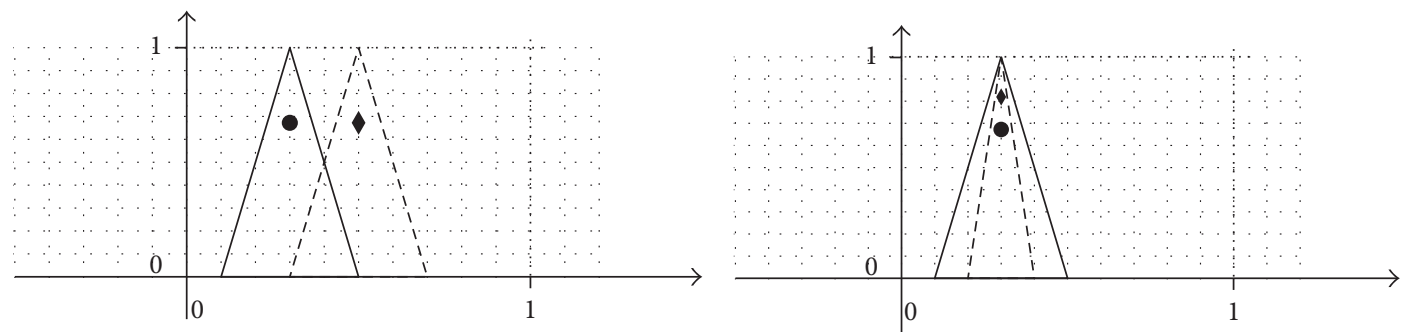

Set 1

$$
\tilde{a}=(0.1,0.3,0.5)_{T}
$$$$
\text { - - } \tilde{b}=(0.3,0.5,0.7)_{T}
$$

- $N_{\tilde{a}}=(0.3000,0.6721)$

- $N_{\tilde{b}}=(0.5000,0.6721)$

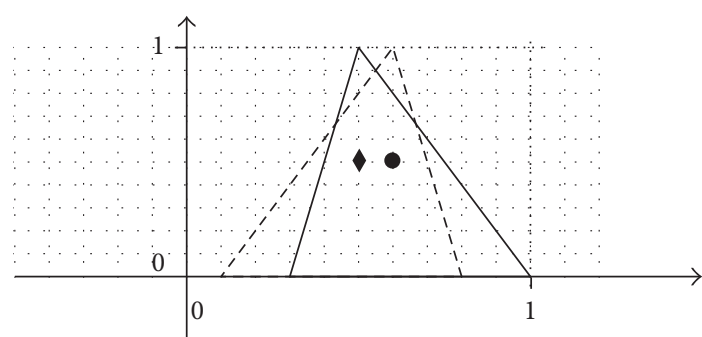

Set 3

$\tilde{a}=(0.3,0.5,1.0)_{T}$

$--\tilde{b}=(0.1,0.6,0.8)_{T}$

$$
\text { - } N_{\tilde{a}}=(0.5983,0.5067)
$$

- $N_{\tilde{b}}=(0.5021,0.5067)$

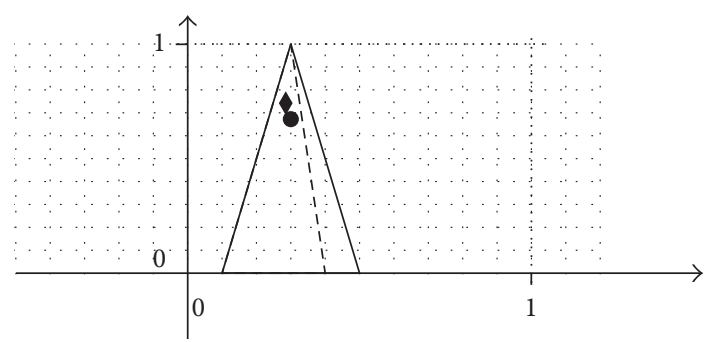

Set 5

$-\tilde{a}=(0.1,0.3,0.5)_{T}$ $--\tilde{b}=(0.1,0.3,0.4)_{T}$
Set 2
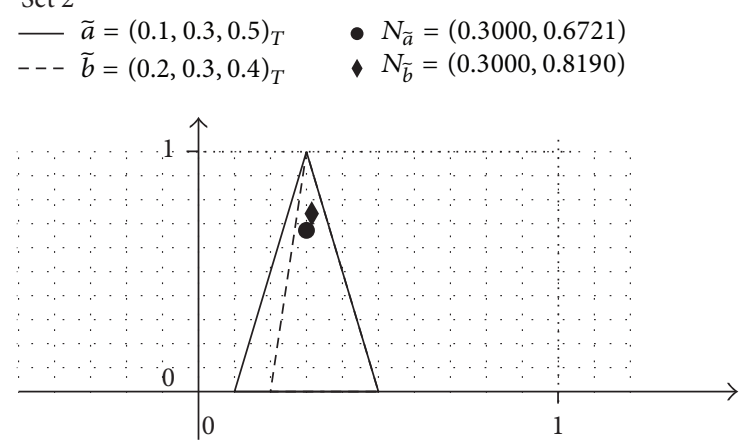

Set 4

$\widetilde{a}=(0.1,0.3,0.5)_{T}$

$--\tilde{b}=(0.2,0.3,0.5)_{T}$

- $N_{\tilde{a}}=(0.3000,0.6721)$

- $N_{\tilde{b}}=(0.3146,0.7419)$

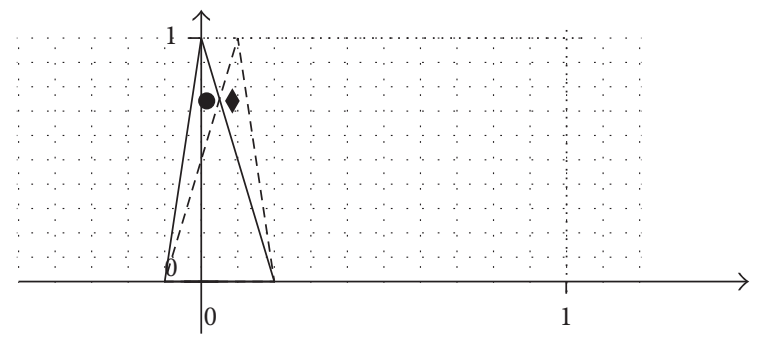

Set 6
$\tilde{a}=(-0.1,0,0.2)_{T}$
$--\tilde{b}=(-0.1,0.1,0.2)_{T}$
- $N_{\tilde{a}}=(0.0148,0.7419)$
- $N_{\tilde{b}}=(0.0852,0.7419)$

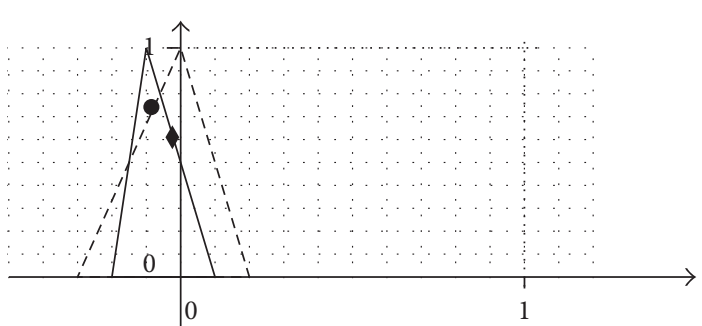

Set 7

$$
\begin{aligned}
& \text { - } \widetilde{a}=(-0.2,-0.1,0.1)_{T} \quad \text { - } N_{\tilde{a}}=(-0.0852,0.7419) \\
& \text { - - } \tilde{b}=(-0.3,0,0.2)_{T} \quad \forall N_{\tilde{b}}=(-0.0242,0.6100)
\end{aligned}
$$

Figure 5: Sets of triangular fuzzy numbers. the method is not discriminative, or it cannot order fuzzy numbers with the same centroid. However, the proposed method avoids these problems for ordering triangular fuzzy numbers. The limitations of each method are presented in Table 2.

\section{Fuzzy Risk Analysis Based on the Proposed Method}

Here, we present an application of the proposed method to a fuzzy risk analysis problem. 
TABLE 1: A comparison of methods.

\begin{tabular}{|c|c|c|c|c|c|c|c|}
\hline \multirow{2}{*}{ Method } & \multicolumn{7}{|c|}{ Ordering results } \\
\hline & Set 1 & Set 2 & Set 3 & Set 4 & Set 5 & Set 6 & Set 7 \\
\hline S.-J. Chen and S.-M. Chen [3] & $\tilde{a} \prec \widetilde{b}$ & $\tilde{a} \prec \tilde{b}$ & $\widetilde{b} \prec \tilde{a}$ & $\tilde{a} \prec \tilde{b}$ & $\tilde{a} \prec \widetilde{b}$ & $\tilde{a} \prec \tilde{b}$ & $\widetilde{b} \prec \widetilde{a}$ \\
\hline Lee and Chen [22] & $\tilde{a} \prec \tilde{b}$ & $\tilde{a} \prec \tilde{b}$ & $\tilde{b} \prec \tilde{a}$ & $\tilde{a} \prec \tilde{b}$ & $\tilde{b} \prec \tilde{a}$ & $\tilde{a} \prec \tilde{b}$ & $\tilde{a}<\tilde{b}$ \\
\hline Akyar et al. [18] & $\tilde{a} \prec \widetilde{b}$ & $\tilde{a} \prec \tilde{b}$ & $\tilde{b} \prec \tilde{a}$ & $\tilde{a} \prec \tilde{b}$ & $\tilde{b} \prec \tilde{a}$ & $\tilde{a} \prec \widetilde{b}$ & $\widetilde{b} \prec \widetilde{a}$ \\
\hline Abbasbandy et al. [15] & $\tilde{a} \prec \tilde{b}$ & $\tilde{b} \prec \tilde{a}$ & $\tilde{b} \prec \tilde{a}$ & $\tilde{a} \prec \tilde{b}$ & $\tilde{b} \prec \tilde{a}$ & $\tilde{a} \prec \tilde{b}$ & $\tilde{b} \prec \tilde{a}$ \\
\hline Chutia et al. [25] & $\tilde{a} \prec \tilde{b}$ & $\tilde{a} \sim \tilde{b}$ & $\tilde{b} \prec \tilde{a}$ & $\tilde{a} \prec \tilde{b}$ & $\tilde{b} \prec \tilde{a}$ & $\tilde{a} \prec \tilde{b}$ & $\tilde{a}<\tilde{b}$ \\
\hline Düzce [21] & $\tilde{a} \prec \widetilde{b}$ & $\tilde{a} \sim \tilde{b}$ & $\tilde{b} \prec \tilde{a}$ & $\tilde{a} \prec \tilde{b}$ & $\tilde{b} \prec \tilde{a}$ & $\tilde{a} \prec \tilde{b}$ & $\tilde{a}<\tilde{b}$ \\
\hline Ezzati et al. [19] & $\tilde{a} \prec \tilde{b}$ & $\tilde{a} \prec \tilde{b}$ & $\tilde{a} \prec \tilde{b}$ & $\tilde{a} \prec \widetilde{b}$ & $\tilde{b} \prec \tilde{a}$ & $\tilde{a} \prec \tilde{b}$ & $\tilde{a}<\widetilde{b}$ \\
\hline Jiang et al. [24] & $\tilde{a} \prec \widetilde{b}$ & $\tilde{a} \prec \tilde{b}$ & $\tilde{b} \prec \tilde{a}$ & $\tilde{a} \prec \tilde{b}$ & $\tilde{b} \prec \tilde{a}$ & $\tilde{a} \prec \tilde{b}$ & $\tilde{a}<\tilde{b}$ \\
\hline Proposed & $\tilde{a} \prec \tilde{b}$ & $\tilde{a} \prec \tilde{b}$ & $\tilde{b} \prec \tilde{a}$ & $\tilde{a} \prec \tilde{b}$ & $\tilde{b} \prec \tilde{a}$ & $\tilde{a} \prec \tilde{b}$ & $\tilde{a} \prec \tilde{b}$ \\
\hline
\end{tabular}

TABle 2: The limitations of each method ( $x$ : does not satisfy the property; $\checkmark$ : satisfies the property).

\begin{tabular}{lccc}
\hline Method & Discriminative & $\begin{array}{c}\text { Rank correctly } \\
\text { FNs with the } \\
\text { same centroid } \\
\text { point }\end{array}$ & $\begin{array}{c}\text { Rank crisp } \\
\text { numbers }\end{array}$ \\
\hline $\begin{array}{l}\text { S.-J. Chen and } \\
\text { S.-M. Chen [3] }\end{array}$ & $\times$ & $\checkmark$ & $\checkmark$ \\
$\begin{array}{l}\text { Lee and Chen } \\
\text { [22] }\end{array}$ & $\checkmark$ & $\checkmark$ & $\checkmark$ \\
Akyar et al. [18] & $\checkmark$ & $\checkmark$ & $\checkmark$ \\
$\begin{array}{l}\text { Abbasbandy et } \\
\text { al. [15] }\end{array}$ & $\times$ & $\times$ & $\checkmark$ \\
Chutia et al. [25] & $\times$ & $\times$ & $\checkmark$ \\
Düzce [21] & $\times$ & $\times$ & $\checkmark$ \\
Ezzati et al. [19] & $\checkmark$ & $\checkmark$ & $\checkmark$ \\
Jiang et al. [24] & $\checkmark$ & $\checkmark$ & $\checkmark$ \\
Proposed & $\checkmark$ & $\checkmark$ & $\checkmark$ \\
\hline
\end{tabular}

Let us assume that we have $n$ manufactories $C_{1}, C_{2}, \ldots, C_{n}$ and manufactory $C_{i}$ produces the component $A_{i}$ which is composed of $m$ different subcomponents $A_{i 1}, A_{i 2}, \ldots, A_{i m}$.

If $\widetilde{r}_{i k}$ denotes the probability of failure and $\widetilde{w}_{i k}$ denotes the severity of loss of the subcomponent $A_{i k}(1 \leq i \leq n$ and $1 \leq k \leq m$ ) then one can obtain the probability of failure $\tilde{r}_{i}$ of the manufactory $C_{i}$. The basic structure used to analyze the probability of failure $\tilde{r}_{i}$ of component $A_{i}$ made by manufactory $C_{i}$ is shown in Figure 6 (see [34]).

We propose a fuzzy risk analysis method similar to that of Chen and Sanguansat (see [17]) as follows.

Step 1. Using the fuzzy weighted method for every component $A_{i}$ made by manufactory $C_{i}$ calculates the probability of failure

$$
\widetilde{r}_{i}=\frac{\left(\sum_{k=1}^{m} \widetilde{r}_{i k} \cdot \widetilde{w}_{i k}\right)}{\sum_{k=1}^{m} \widetilde{w}_{i k}}
$$

$(i=1,2, \ldots, n)$ by means of $\widetilde{r}_{i k}$ and $\widetilde{w}_{i k}$ of subcomponent $A_{i k}$.
TABLE 3: Linguistic terms and their corresponding triangular fuzzy numbers.

\begin{tabular}{lc}
\hline Linguistic term & Triangular fuzzy numbers \\
\hline Very low & $(0,0,0.1)_{T}$ \\
Low & $(0,0.1,0.3)_{T}$ \\
Fairly low & $(0.1,0.3,0.5)_{T}$ \\
Medium & $(0.3,0.5,0.7)_{T}$ \\
Fairly high & $(0.5,0.7,0.9)_{T}$ \\
High & $(0.7,0.9,1)_{T}$ \\
Very high & $(0.9,1,1)_{T}$ \\
\hline
\end{tabular}

Step 2. Transform each triangular fuzzy number $\tilde{r}_{i}=\left(r_{i 1}, r_{i 2}\right.$, $\left.r_{i 3}\right)_{T}$ into standardized triangular fuzzy number

$$
\tilde{r}_{i}^{*}=\left(\frac{r_{i 1}}{k}, \frac{r_{i 2}}{k}, \frac{r_{i 3}}{k}\right)_{T},
$$

where $k=\max \left\{\left\lceil\left|r_{i j}\right|\right\rceil, 1\right\}, i=1,2, \ldots, n, j=1,2,3$, and $\left\lceil\left|r_{i j}\right|\right\rceil$ denotes the smallest integer greater than the absolute value of the real number $r_{i j}$.

Step 3. For all $i=1,2, \ldots, n$ order triangular fuzzy numbers $\tilde{r}_{i}^{*}$ by the proposed method. The larger triangular fuzzy number represents the higher probability of failure of component $A_{i}$ made by the manufactory $C_{i}$.

Here, we present an example to illustrate the fuzzy risk analysis algorithm inspired by [16].

Example 6. Let us assume that $A_{1}, A_{2}$, and $A_{3}$ are the same product manufactured by different manufactories $C_{1}, C_{2}$, and $C_{3}$, respectively. Furthermore, for all $i=1,2,3$ the component $A_{i}$ is composed of three subcomponents $A_{i 1}, A_{i 2}$, and $A_{i 3}$.

Using $\widetilde{w}_{i k}$, the severity of loss of subcomponent $A_{i k}$, and $\tilde{r}_{i k}$, which is the probability of failure of subcomponent $A_{i k}$, we can find the probability of failure $\widetilde{r}_{i}$ of component $A_{i}$ made by manufactory $C_{i}$.

We use a seven-member linguistic term set shown in Table 3, suggested by S.-J. Chen and S. M. Chen, to represent the linguistic terms and their corresponding triangular fuzzy numbers (see [9]). 
TABLE 4: Linguistic values of the evaluating items of the subcomponents made by manufactories.

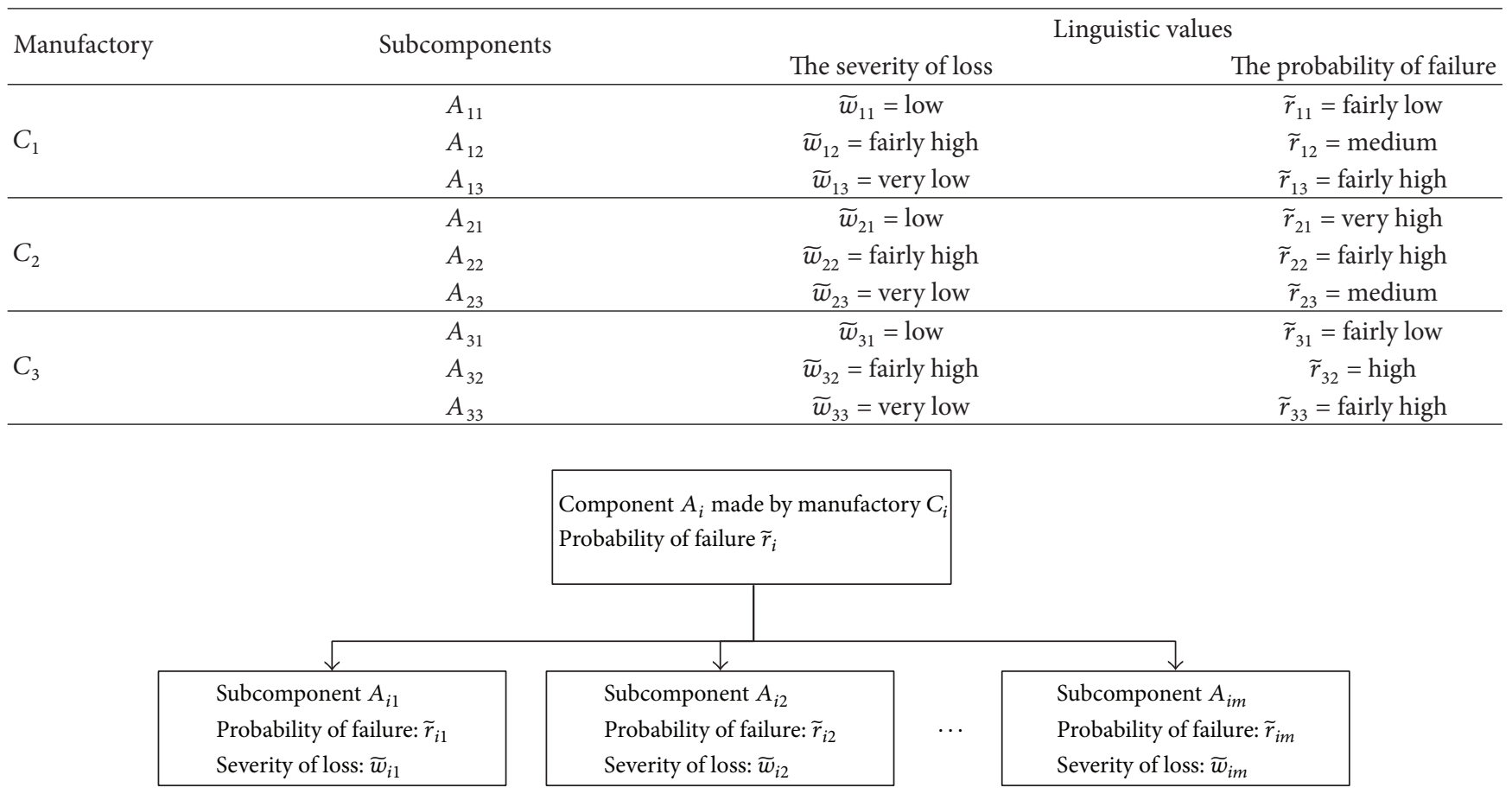

FIgURE 6: The structure for fuzzy risk analysis.

The linguistic values of $\widetilde{r}_{i k}$ and $\widetilde{w}_{i k}$ of subcomponent $A_{i k}$ made by manufactory $C_{i}$ are shown in Table 4.

Step 1. From (26) and the given data we get

$$
\begin{aligned}
& \tilde{r}_{1}=(0.3,0.475,0.6692)_{T}, \\
& \tilde{r}_{2}=(0.5,0.7375,0.9077)_{T}, \\
& \tilde{r}_{3}=(0.7,0.825,0.8769)_{T} .
\end{aligned}
$$

Step 2. Since $k=\max \left\{\left\lceil\left|r_{i j}\right|\right\rceil, 1\right\}=1$ for all $i=1,2, \ldots, 3$ and $j=1,2,3$, it is not necessary to transform triangular fuzzy numbers $\widetilde{r}_{i}$ into standardized form. Therefore we get

$$
\begin{aligned}
& \tilde{r}_{1}^{*}=\widetilde{r}_{1}=(0.3,0.475,0.6692)_{T}, \\
& \widetilde{r}_{2}^{*}=\widetilde{r}_{2}=(0.5,0.7375,0.9077)_{T}, \\
& \widetilde{r}_{3}^{*}=\widetilde{r}_{3}=(0.7,0.825,0.8769)_{T} .
\end{aligned}
$$

Step 3. It follows from (16) that

$$
\begin{aligned}
& \mathcal{N}\left(\widetilde{r}_{1}^{*}\right)=(0.4784,0.6928,0.475) \\
& \mathcal{N}\left(\widetilde{r}_{2}^{*}\right)=(0.7237,0.6669,0.7375) \\
& \mathcal{N}\left(\widetilde{r}_{3}^{*}\right)=(0.8188,0.8389,0.825)
\end{aligned}
$$

Since $\mathcal{N}\left(\widetilde{r}_{1}^{*}\right)<_{L} \mathcal{N}\left(\widetilde{r}_{2}^{*}\right)<_{L} \mathcal{N}\left(\widetilde{r}_{3}^{*}\right)$ then $\widetilde{r}_{1}^{*} \prec \widetilde{r}_{2}^{*} \prec \widetilde{r}_{3}^{*}$. This means that component $A_{3}$, made by manufactory $C_{3}$, has the highest probability of failure, followed by $C_{2}$ and $C_{1}$, respectively.

\section{Conclusion}

In this paper, we present a new method for ordering triangular fuzzy numbers. The Nagel point of a triangle and the peak point for ordering triangular fuzzy numbers are used. We show that the proposed method can overcome the drawbacks of certain well-known ordering and ranking methods. The advantages of the proposed method are presented with comparative examples. We also give an application of the proposed fuzzy ordering method to the fuzzy risk analysis problem, where the evaluating values are represented by triangular numbers. Consequently, the proposed ordering process is simple and efficient for calculation and comparison purposes, which makes its application more effective.

\section{Competing Interests}

The author declares that she has no competing interests.

\section{Acknowledgments}

This study was supported by the Anadolu University Scientific Research Projects Commission under Grant no. 1403 F077.

\section{References}

[1] E. Akyar, "A fictitious play algorithm for matrix games with fuzzy payoffs," Abstract and Applied Analysis, vol. 2012, Article ID 950482, 12 pages, 2012. 
[2] E. Akyar, H. Akyar, and S. A. Düzce, "Fuzzy risk analysis based on a geometric ranking method for generalized trapezoidal fuzzy numbers," Journal of Intelligent and Fuzzy Systems, vol. 25, no. 1, pp. 209-217, 2013.

[3] S.-J. Chen and S.-M. Chen, "Fuzzy risk analysis based on the ranking of generalized trapezoidal fuzzy numbers," Applied Intelligence, vol. 26, no. 1, pp. 1-11, 2007.

[4] A. Marszalek and T. Burczynski, "Modeling and forecasting financial time series with ordered fuzzy candlesticks," Information Sciences, vol. 273, pp. 144-155, 2014.

[5] N. Xie and J. Xin, "Interval grey numbers based multi-attribute decision making method for supplier selection," Information Sciences, vol. 43, pp. 1064-1078, 2014.

[6] W. Yue, Y. Cai, Q. Rong, C. Li, and L. Ren, "A hybrid lifecycle and fuzzy-set-pair analyses approach for comprehensively evaluating impacts of industrial wastewater under uncertainty," Journal of Cleaner Production, vol. 80, pp. 57-68, 2014.

[7] M. Barajas and B. Agard, "Improved fuzzy ranking procedure for decision making in product design," International Journal of Production Research, vol. 48, no. 18, pp. 5433-5453, 2010.

[8] K.-M. Björk, "An analytical solution to a fuzzy economic order quantity problem," International Journal of Approximate Reasoning, vol. 50, no. 3, pp. 485-493, 2009.

[9] S.-J. Chen and S.-M. Chen, "Fuzzy risk analysis based on similarity measures of generalized fuzzy numbers," IEEE Transactions on Fuzzy Systems, vol. 11, no. 1, pp. 45-56, 2003.

[10] S. Murakami, S. Maeda, and S. Imamura, "Fuzzy decision analysis on the development of centralized regional energy control system," in Proceedings of the IFAC Conference on Fuzzy Information, Knowledge Representation and Decision Analysis, pp. 363-368, Pergamon, Marseille, France, July 1983.

[11] R. Jain, "Decision-making in the presence of fuzzy variables," IEEE Transactions on Systems, Man and Cybernetics, vol. 6, no. 10, pp. 698-703, 1976.

[12] R. R. Yager, “On a general class of fuzzy connectives," Fuzzy Sets and Systems, vol. 4, no. 3, pp. 235-242, 1980.

[13] C.-H. Cheng, "A new approach for ranking fuzzy numbers by distance method," Fuzzy Sets and Systems, vol. 95, no. 3, pp. 307317, 1998.

[14] S. Abbasbandy and B. Asady, "Ranking of fuzzy numbers by sign distance," Information Sciences, vol. 176, no. 16, pp. 2405-2416, 2006.

[15] S. Abbasbandy, R. Nuraei, and M. Ghanbari, "Revision of sign distance method for ranking of fuzzy numbers," Iranian Journal of Fuzzy Systems, vol. 10, no. 4, pp. 101-117, 151, 2013.

[16] S.-M. Chen and C.-H. Wang, "Fuzzy risk analysis based on ranking fuzzy numbers using $\alpha$-cuts, belief features and signal/noise ratios," Expert Systems with Applications, vol. 36, no. 3, pp. 5576-5581, 2009.

[17] S.-M. Chen and K. Sanguansat, "Analyzing fuzzy risk based on a new fuzzy ranking method between generalized fuzzy numbers," Expert Systems with Applications, vol. 38, no. 3, pp. 2163-2171, 2011.

[18] E. Akyar, H. Akyar, and S. A. Düzce, "A new method for ranking triangular fuzzy numbers," International Journal of Uncertainty, Fuzziness and Knowledge-Based Systems, vol. 20, no. 5, pp. 729740, 2012.

[19] R. Ezzati, S. Khezerloo, and S. Ziari, "Application of parametric form for ranking of fuzzy numbers," Iranian Journal of Fuzzy Systems, vol. 12, no. 1, pp. 59-74, 136, 2015.
[20] R. Ezzati, T. Allahviranloo, S. Khezerloo, and M. Khezerloo, "An approach for ranking of fuzzy numbers," Expert Systems with Applications, vol. 39, no. 1, pp. 690-695, 2012.

[21] S. A. Düzce, "A new ranking method for trapezial fuzzy numbers and its application to fuzzy risk analysis," Journal of Intelligent \& Fuzzy Systems, vol. 28, no. 3, pp. 1411-1419, 2015.

[22] L.-W. Lee and S.-M. Chen, "Fuzzy risk analysis based on fuzzy numbers with different shapes and different deviations," Expert Systems with Applications, vol. 34, no. 4, pp. 2763-2771, 2008.

[23] S. Abbasbandy and T. Hajjari, "A new approach for ranking of trapezoidal fuzzy numbers," Computers \& Mathematics with Applications, vol. 57, no. 3, pp. 413-419, 2009.

[24] W. Jiang, Y. Luo, X.-Y. Qin, and J. Zhan, "An improved method to rank generalized fuzzy numbers with different left heights and right heights," Journal of Intelligent \& Fuzzy Systems. Applications in Engineering and Technology, vol. 28, no. 5, pp. 2343-2355, 2015.

[25] R. Chutia, R. Gogoi, and D. Datta, "Ranking $p$-norm generalised fuzzy numbers with different left height and right height using integral values," Mathematical Sciences, vol. 9, no. 1, pp. 1-9, 2015.

[26] R. Nazirah and M. Daud, "A comparative analysis of centroid methods in ranking fuzzy numbers," European Journal of Scientific Research, vol. 28, no. 3, pp. 492-501, 2009.

[27] C. Kimberling, "Central points and central lines in the plane of a triangle," Mathematics Magazine, vol. 67, no. 3, pp. 163-187, 1994.

[28] B. Bede, Mathematics of Fuzzy Sets and Fuzzy Logic, vol. 295 of Studies in Fuzziness and Soft Computing, Springer, Heidelberg, Germany, 2013.

[29] K. H. Lee, First Course on Fuzzy Theory and Applications, Springer, Heidelberg, Germany, 2005.

[30] G. E. Martin, Transformation Geometry: An Introduction to Symmetry, Springer, New York, NY, USA, 1982.

[31] G. E. Gallatly, Modern Geometry of the Triangle, Hodgson, London, UK, 2nd edition, 1913.

[32] X. Wang and E. E. Kerre, "Reasonable properties for the ordering of fuzzy quantities. I," Fuzzy Sets and Systems, vol. 118, no. 3, pp. 375-385, 2001.

[33] X. Wang and E. E. Kerre, "Reasonable properties for the ordering of fuzzy quantities. II," Fuzzy Sets and Systems, vol. 118, no. 3, pp. 387-405, 2001.

[34] K. J. Schmucker, Fuzzy Sets, Natural Language Computations, and Risk Analysis, Computer Science Press, Rockville, Md, USA, 1984. 


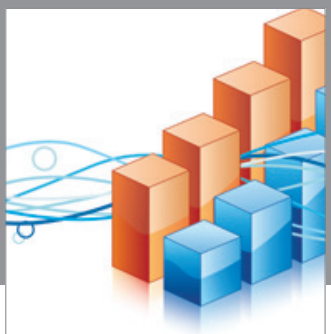

Advances in

Operations Research

vatem alat4

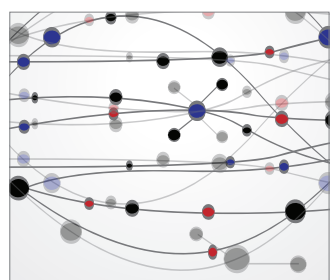

\section{The Scientific} World Journal
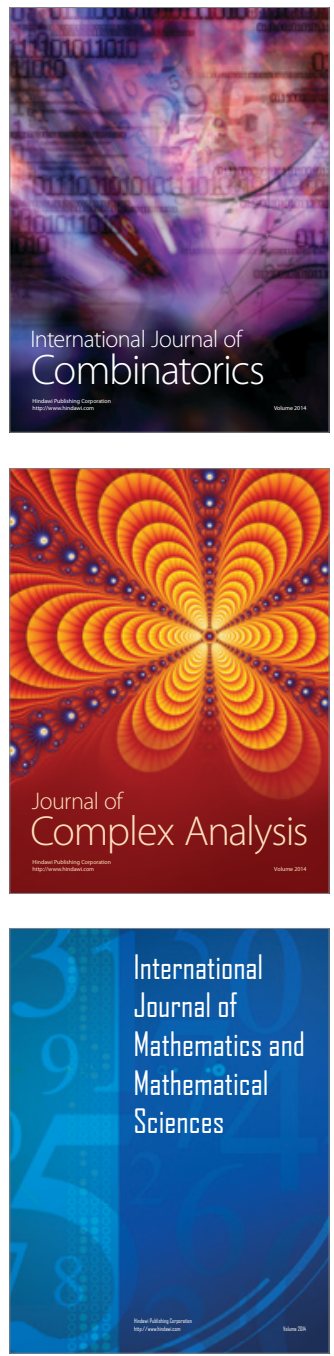
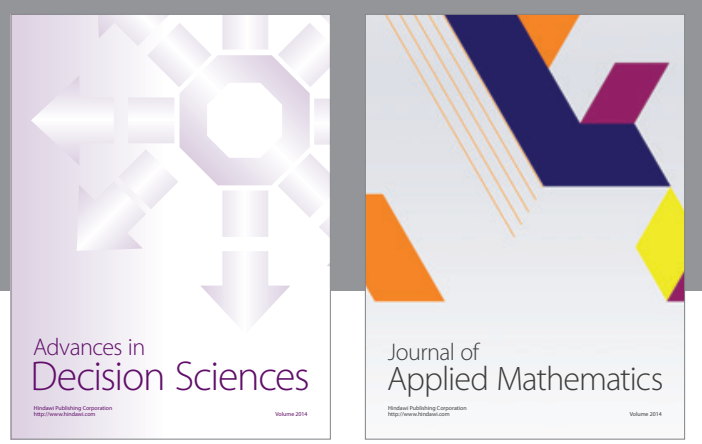

Algebra

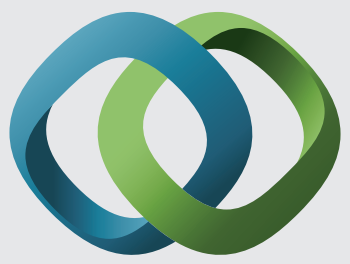

\section{Hindawi}

Submit your manuscripts at

http://www.hindawi.com
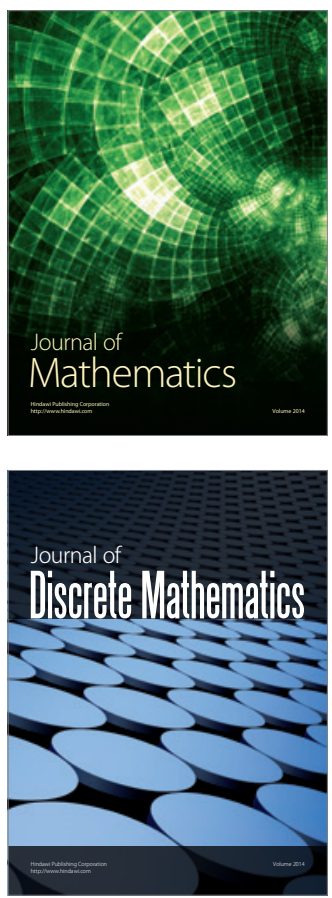

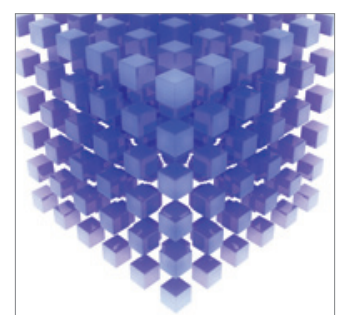

Mathematical Problems in Engineering
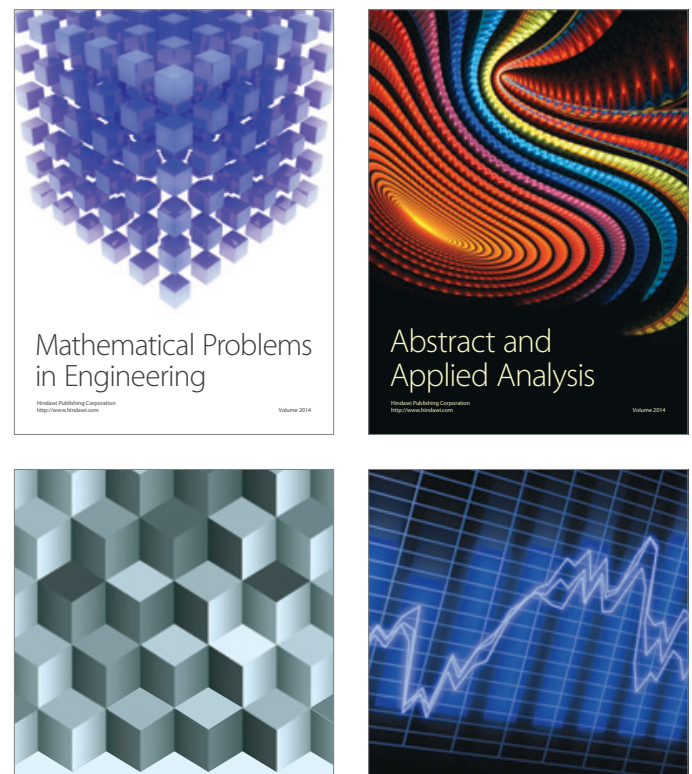

Journal of

Function Spaces

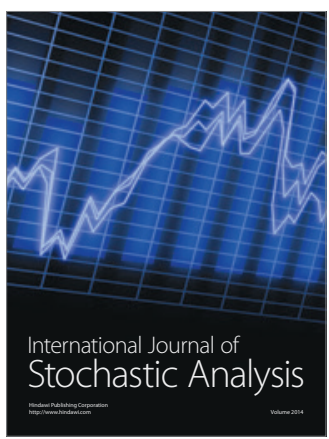

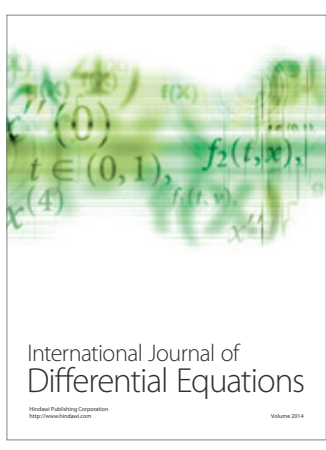
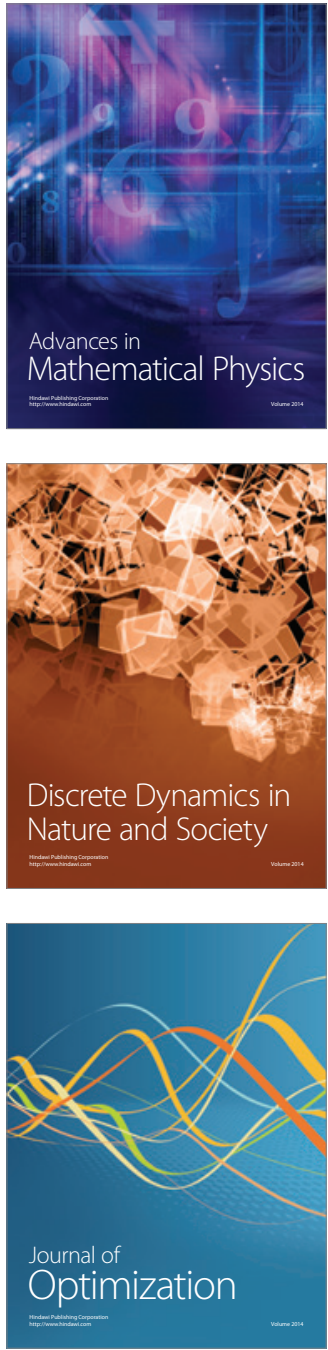